\title{
ANÁLISES ESTATÍSTICAS DAS TENDÊNCIAS DE ELEVAÇÃO NAS SÉRIES ANUAIS DE TEMPERATURA MÍNIMA DO AR NO ESTADO DE SÃO PAULO $\left({ }^{1}\right)$
}

\author{
GABRIEL CONSTANTINO BLAIN $\left(\left(^{*}\right)\right.$; MICHELLE CRISTINA ARAUJO PICOLI $\left({ }^{3}\right)$; JORGE LULU $\left({ }^{4}\right)$
}

\begin{abstract}
RESUMO
Considerando que algumas investigações sobre o tema aquecimento global utilizam longas séries temporais de temperatura uma questão que deve ser levantada é relativa a influência de possíveis concentrações de fontes de calor urbano a que os postos meteorológicos possam estar submetidos. O primeiro passo para responder a essa questão é verificar se, em uma mesma região, ocorrem tendências concomitantes de elevação nos dados de temperatura em diversas localidades, possivelmente ligadas a fenômenos de escala global. Entretanto, se essa elevação tiver seu início em períodos bastante distintos entre as diversas localidades, o descarte de forçantes radioativas locais como principais responsáveis por esse aumento, dificilmente poderá ser realizado. O objetivo do trabalho foi detectar tendências de elevação nas séries anuais de temperatura mínima, bem como a data inicial dessas prováveis alterações, em seis localidades do Estado de São Paulo. A caracterização das séries foi realizada por meio da distribuição normal. O teste de Mann-Kendall não indicou tendências concomitantes nos dados anuais de temperatura mínima. Dessa forma, fatores de escala local parecem se sobrepor a fatores de escala global como principais forçantes radioativas causadoras do aumento médio dos valores da variável meteorológica sob investigação. Os resultados demonstram a necessidade de avaliar e/ou isolar fatores locais, como a urbanização, antes que investigações sobre os temas aquecimento global e zoneamento agrícola sejam realizadas com base em séries históricas no Estado de São Paulo.
\end{abstract}

Palavras-chave: Mann-Kendall, urbanização, ilha de calor.

\section{ABSTRACT \\ STATISTICAL ANALYSIS OF ANNUAL MINIMUM AIR TEMPERATURE TRENDS IN SÃO PAULO STATE}

Considering that some global warming investigations are based on historical series of air temperature, a question that should be raised is about the influence of urban heat sources on the weather stations areas. The first approach to answering this question is to verify if different weather stations that are geographically close to each others, show elevation trends starting at the same time, possible related to global radiative forcing. However, if these trends start at quite different period among the several weather stations, the elimination of local radiative forcing as the main responsible for this trend is not possible. The aim of this work was to detect continuous trends in annual minimum air temperature series of six weather stations of the State of São Paulo, Brazil. The initial date of those trends was also evaluated. The series were fitted to the normal distribution. The Mann-Kendall test did not detected elevation trends starting at the same time among the analyzed series. Thus, local radiative forcing seems to be a more important factor, responsible for the average increase of the meteorological variable under investigation, than global radiative forcing. The results also indicate that local factors, such as urbanization influences, have to be evaluated and isolated in global warming or agricultural zoning investigations based on minimum air temperature series of the State of São Paulo, Brazil.

Key words: Mann-Kendall test, air temperature urbanization influences, urban heat island

(1) Recebido para publicação em 28 de abril de 2008, aceito em

( $\left.{ }^{2}\right)$ Autor correspondente: Instituto Agronômico de Campinas (IAC/APTA/SAA), Caixa Postal 28, 13001-970 Campinas (SP), E-mail: gabriel@iac.sp.gov.br $\left(^{*}\right)$ Autor correspondente.

$\left({ }^{3}\right)$ Universidade Estadual de Campinas, Faculdade de Engenharia Agrícola, Campinas (SP), Brasil.

$\left({ }^{4}\right)$ Escola Superior de Agricultura "Luiz de Queiroz" /USP, Departamento de Ciências Exatas, Piracicaba, SP, Brasil. 


\section{INTRODUÇÃO}

De acordo com o IPCC (2007), o aumento médio da temperatura do ar atmosférico é inequívoco, e onze dos doze anos anteriores a 2006 estão entre os anos mais quentes do período de 1850, início da medição da temperatura do ar por aparelhos, a 2006. Ainda, segundo esse relatório, foi observado na temperatura média global aumento linear de aproximadamente 0,13 ${ }^{\circ} \mathrm{C}$ por década nos últimos 50 anos (1957 a 2006). Esse aumento é aproximadamente duas vezes superior ao observado no período de 1850 a 1956.

A principal forçante radioativa $\left({ }^{5}\right)$ desse fenômeno é a ação antropogênica estimada em 1,6 W $\mathrm{m}^{-2}$ com intervalo de confiança entre 1,49 a $1,83 \mathrm{~W} \mathrm{~m}$ ${ }^{2}$. O processo mais importante dessa forçante é a queima de combustíveis fósseis que tem elevado a concentração do $\mathrm{CO}_{2}$ atmosférico. É interessante ressaltar que o IPCC (2007) afirma que as ilhas de calor relativas ao clima urbano são reais, mas possuem efeitos desprezíveis (negligible) e apenas locais. É admitido que sua influência no aumento da temperatura média atmosférica global é menor que $0,006^{\circ} \mathrm{C}$ a cada década.

De acordo com OKE (1987), a alta concentração de fontes de calor nas cidades resulta no desenvolvimento das ilhas de calor urbano. ICHINOSE et al. (1999), em um estudo realizado na região metropolitana de Tóquio, Japão, afirma que a taxa de evapotranspiração, tipicamente mais baixa na cidade, acentua ainda mais o contraste de temperatura com suas redondezas. De acordo com LOMBARDO (1984), a intensidade de uma ilha de calor é altamente influenciada pelas condições sinóticas atuantes. Os maiores gradientes de temperatura observados entre a área urbana da cidade de São Paulo e as áreas rurais podem atingir valores superiores a $10^{\circ} \mathrm{C}$ no inverno.

Segundo Freitas e Silva Dias (2005), a altura da CLP (camada limite planetária) é altamente modificada pela presença de urbanização, sendo cerca de $150 \mathrm{~m}$ mais alta do que seria no caso de uma zona vegetada em dias quando as fontes antropogênicas são menos intensas. Quando a participação dessas fontes é mais efetiva, a diferença pode ultrapassar 400 $\mathrm{m}$. A CLP pode ser influenciada até mesmo em regiões relativamente distantes das áreas urbanas.

$\left({ }^{5}\right)$ Medida da influência de um fator no que diz respeito à alteração do balanço de energia do sistema superfície -atmosfera. Pode ser entendido como um índice que exprime a importância de um processo como mecanismo potencial de alterações climáticas.
Comparações entre a área urbana e suas vizinhanças indicam que durante o período diurno essas diferenças variam entre 200 e $600 \mathrm{~m}$, dependendo da região. De acordo com Freitas e SiLVADiAs (2005), essas diferenças podem ser explicadas pela alteração do balanço de energia na superfície. $\mathrm{Na}$ região urbana verificam-se altos valores para a razão de Bowen, em contraste com outras regiões relativamente distantes da área metropolitana, onde os fluxos de calor sensível e latente são equivalentes. Esses autores afirmam também que as diferenças de temperatura observadas entre as áreas urbanas e suas vizinhanças são altamente dependentes de suas dimensões, sendo em regiões urbanas relativamente pequenas o efeito de ilha de calor pode ser imperceptível em consequência da rápida mistura com $\mathrm{o}$ ar das regiões vizinhas.

Analisando-se as afirmações descritas, tornase evidente que apesar do fenômeno "ilhas de calor" não poder ser considerado uma forçante radioativa de escala global, sua forte influência no clima local de uma região metropolitana e, consequentemente, nos registros de temperatura do ar realizados em postos meteorológicos próximos a centros urbanos não pode ser descartada. Dessa forma, considerando que algumas investigações sobre o tema aquecimento global utilizam tais séries, a detecções de tendências de aumento nos valores de temperatura do ar, ocorrido em anos recentes, deve responder a perguntas como: A principal forçante radioativa dessa elevação é relativa a fenômenos de escala global ou a modificações locais, como por exemplo, a urbanização, da região em que o posto foi instalado.

O primeiro passo para responder essa questão é verificar se, em uma dada região (Estado de São Paulo, por exemplo) essa tendência contínua de elevação nos dados de temperatura ocorre concomitantemente em diversas localidades da região considerada estando, possivelmente, ligada a fenômenos de escala global. Se, por outro lado, esse aumento tiver seu início em períodos bastante distintos entre as diversas localidades, o descarte de forçantes radioativas locais como principais responsáveis por esse aumento torna-se bastante difícil.

BLAIN et al. (2007), por meio de análise de paralelismo e coincidência de retas geradas por regressão linear simples, indicou a existência de aumento linear na série de temperatura mínima de Campinas (SP). Esse modelo explicou aproximadamente $77 \%$ da variabilidade dessa variável meteorológica entre o período de 1890 e 2005. Apesar dessa constatação, os autores não indicam o período de início dessa tendência e por trabalhar com apenas um local, não verificaram se esse aumento ocorreu em outras regiões do Estado 
de São Paulo. Na série de temperatura máxima não houve tendências contínuas de elevação.

Dessa forma, o objetivo do trabalho foi caracterizar as séries anuais de temperatura mínima (Tmín) em seis localidades do Estado de São Paulo, detectando possíveis tendências contínuas de elevação. A data inicial dessas possíveis elevações também foi avaliada. Espera-se que essa investigação forneça importantes informações estatísticas a respeito do tema mudanças climáticas.

\section{MATERIAL E MÉTODOS}

Foram utilizados dados anuais de temperatura mínima do ar, pertencentes à Secretaria de Agricultura e Abastecimento do Estado de São Paulo, das localidades de Cordeirópolis/Limeira $\left(22^{\circ} 27^{\prime} \mathrm{S}\right.$; $47^{\circ} 24^{\prime} \mathrm{W}$; $639 \mathrm{~m}$; posto situado no limite entre os dois municípios), Monte Alegre do Sul $\left(22^{\circ} 42^{\prime} \mathrm{S} ; 46^{\circ} 39^{\prime} \mathrm{W}\right.$; $777 \mathrm{~m})$, Ribeirão Preto $\left(21^{\circ} 11^{\prime} \mathrm{S} ; 47^{\circ} 48^{\prime} \mathrm{W} ; 620 \mathrm{~m}\right)$, Pindorama $\left(21^{\circ} 13^{\prime} \mathrm{S} ; 48^{\circ} 54^{\prime} \mathrm{W} ; 562 \mathrm{~m}\right)$ e Piracicaba $\left(22^{\circ} 42^{\prime} \mathrm{S} ; 47^{\circ} 38^{\prime} \mathrm{W} ; 546 \mathrm{~m}\right.$; pertencente à Escola Superior de Agricultura "Luiz de Queiroz" ESALQ/USP). De acordo com Mello et al. (1994), a série do Município de Campinas contém, no ano de 1956, uma alteração no local de coleta $\left(22^{\circ} 53^{\prime} \mathrm{S}, 47^{\circ} 05^{\prime} \mathrm{W}\right.$ e $663 \mathrm{~m}$ para $22^{\circ} 54^{\prime} \mathrm{S} 47^{\circ} 05^{\prime} \mathrm{W}$ e $674 \mathrm{~m}$, com aproximadamente três quilômetros de distância em linha reta). Entretanto, segundo esses autores, os dados de temperatura mínima relativos aos períodos anterior e posterior a essa mudança de local podem ser considerados oriundos de uma mesma população. Nas demais localidades, não há, na literatura, indicações de significativas alterações de locais de coleta. Assim, trabalhos como os de CAMARgo et al. (1993), AstolPho et al. (2004) e SANSIGOlO (2008) indicam que as séries utilizadas nesse estudo também podem, dentro de cada localidade, serem consideradas homogêneas.

Os fatores principais de escolha dessas localidades foram a disponibilidade, a confiabilidade e a distribuição geográfica das séries históricas. A disponibilidade de dados permitiu, nas localidades de Campinas e Piracicaba, a adoção do período homogêneo de 1917 a 2006. Nas demais, os anos de 1951 a 2006 foram utilizados. Na figura 1 são ilustradas localização das estações utilizadas.

As regiões ilustradas na Figura 1 representam os tipos climáticos, de acordo com a classificação de Köeppen, espacialmente preponderantes no Estado, sendo eles: Cfa para as regiões de Campinas, Cordeirópolis/Limeira e Monte Alegre do Sul, Aw para as regiões de Ribeirão Preto, Pindorama e Piracicaba, conforme descrito em Rolim et al. (2007). Na tabela 1 é relacionado o número de habitantes das localidades em estudo fornecidos pelo Instituto Nacional de Geografia e Estatística (IBGE), em agosto de $2006\left(^{6}\right)$.

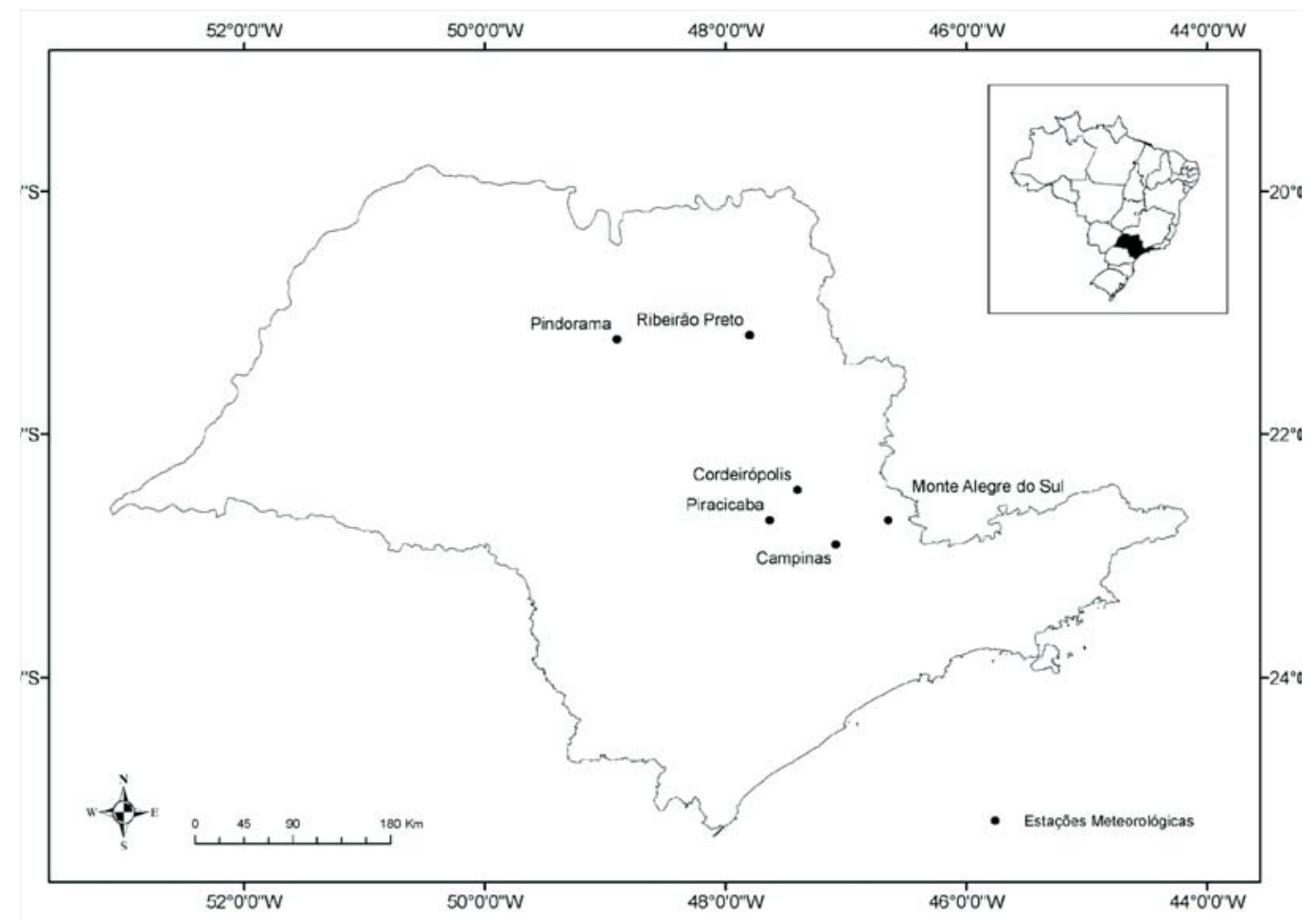

Figura 1. Distribuição espacial das estações meteorológicas do Estado de São Paulo utilizadas no estudo. 
Tabela 1. Número de habitantes dos municípios de Campinas, Cordeirópolis/Limeira, Monte Alegre do Sul, Pindorama, Piracicaba e Ribeirão Preto, Estado de São Paulo, Brasil.

\begin{tabular}{lc}
\hline Região & Número de habitantes \\
\hline Campinas & 1.059 .420 \\
Cordeirópolis / Limeira & 298.863 \\
Monte Alegre do Sul & 6.973 \\
Pindorama & 13.652 \\
Piracicaba & 366.442 \\
Ribeirão Preto & 559.650 \\
\hline
\end{tabular}

A fim de avaliar possíveis mudanças temporais na distribuição de frequência de ocorrência de valores de Tmín, as séries de Campinas e Piracicaba foram divididas em três períodos ou amostras de 30 anos: 1917 a 1946 (P1), 1947 a 1976 (P2) e 1977 a 2006 (P3), os quais foram determinados com base na série disponível de dados e nas recomendações da Organização Meteorológica Mundial, quanto ao intervalo de tempo mínimo necessário, 30 anos, para a caracterização climática de uma região. Após essa etapa, P1, P2 e P3 foram, dentro de cada localidade, comparados estimando-se a função de densidade de probabilidade normal (FDPN) e seus parâmetros (média aritmética ì e desvio padrão ó) de cada período. Uma variável aleatória $X$ å $R$, tem distribuição normal (DN) se sua função de densidade de probabilidade assumir a forma:

$$
f(X)=\frac{1}{\sqrt{2 \pi} \sigma} \exp \left[-\frac{1}{2}\left(\frac{X-\mu}{\sigma}\right)\right]
$$

Segundo Morettin e Bussab (2003), a DN é uma das mais importantes distribuições, pois muitas técnicas estatísticas, tais como análises de variância, regressão e alguns testes de hipóteses assumem a normalidade dos dados.

O grau de aderência das séries de Tmín à DN foi avaliado por meio do teste de Kolmogorov-Smirnov (KS), descrito em Assis et al. (1996). Assis et al. (2004), ao avaliarem o ajuste de séries históricas de temperatura e radiação solar global diária às funções de densidade de probabilidade normal e log-normal, concluíram que a distribuição normal representa muito bem a variabilidade temporal diária da temperatura e da radiação solar global em Piracicaba$\mathrm{SP}$. Os autores afirmam também que o teste de aderência de KS é bastante recomendado para o caso de séries com dados aproximadamente simétricos.

O mesmo método foi empregado nas demais localidades, porém devido à menor disponibilidade de dados foram utilizados apenas os períodos de 1951 a 1978 e 1979 a 2006.
Segundo Goossens e Berger (1986), o teste de Mann-Kendall (MK) é apropriado para detectar alterações de ordem climática em séries meteorológicas, permitindo, também, a localização aproximada do ponto inicial dessa alteração. Proposto inicialmente por SNEYERS (1975), o MK considera que, na hipótese de estabilidade de uma série temporal, a sucessão de valores ocorre de forma independente e a distribuição de probabilidade deve permanecer sempre a mesma caracterizando uma série aleatória simples. MORAEs et al. (1995) descrevem o método considerando uma série temporal $\mathrm{Xi}$ de $\mathrm{N}$ termos. $\mathrm{O}$ teste consiste na soma tn do número de termos mi da série, relativo ao valor $\mathrm{Xi}$ cujos termos precedentes $\mathrm{Xj}$ são inferiores ao mesmo, isto é, $\mathrm{Xj}<\mathrm{Xi}$ :

$$
\text { tn }=\sum_{\mathrm{i}=1}^{\mathrm{N}} \mathrm{mi}
$$

Para séries com grande número de termos, sob a hipótese nula (Ho) de ausência de tendência, tn apresentará distribuição normal com média e variância:

$$
\begin{aligned}
& \mathrm{E}(\mathrm{tn})=\mathrm{N}(\mathrm{N}-1) / 4 \\
& \operatorname{Var}(\mathrm{tn})=\mathrm{N}(\mathrm{N}-1)(2 \mathrm{~N}+5) / 72
\end{aligned}
$$

A significância estatística de tn para Ho pode ser avaliada por meio de um teste bilateral estimandose a estatística $\mathrm{u}(\mathrm{tn})$ :

$$
\mathrm{u}(\mathrm{tn})=\frac{\mathrm{tn}-\mathrm{E}(\mathrm{tn})}{\sqrt{\operatorname{var}(\mathrm{tn})}}
$$

O valor da probabilidade $p_{1}$ é calculada por meio da tabela da normal reduzida, tal que $\mathrm{p}_{1}=$ $\operatorname{prob}(|\mathrm{u}|>|\mathrm{u}(\mathrm{tn})|)$. Sendo $\mathrm{p}_{0} \mathrm{o}$ nível de significância do teste, Ho não é aceita se $\mathrm{p}_{1}>\mathrm{p}_{0}$. A rejeição de Ho indica a existência de tendência significativa; o sinal da estatística $\mathrm{u}(\mathrm{tn})$ indica se a tendência é positiva $(\mathrm{u}(\mathrm{tn})>0)$ ou negativa $(\mathrm{u}(\mathrm{tn})<0)$.

Em sua versão sequencial, a equação 5 é calculada no sentido direto da série, partindo do valor de $\mathrm{i}=1$ até $\mathrm{i}=\mathrm{N}$, gerando a estatística $\mathrm{u}(\mathrm{tn})$, e no sentido inverso da série, partindo do valor de $\mathrm{i}=\mathrm{N}$ até $i=1$, gerando a estatística $u^{*}(t n)$. A interseção das duas curvas geradas representa o ponto aproximado de uma mudança brusca na média. Se essa intersecção ocorrer fora do intervalo de confiança $-1,96<\mathrm{u}(\mathrm{tn})<$ 1,96 , a tendência é significativa e Ho é rejeitada à $5 \%$ de significância. Análises de regressão linear simples também foram realizadas.

\section{RESULTADOS E DISCUSSÃO}

Apesar de serem apresentadas algumas inferências sobre possíveis forçantes radioativas e sua 
possível relação com a tendência temporal dos dados de Tmín, é importante ressaltar que as análises matemáticas/estatísticas empregadas no estudo são conclusivas apenas sob o ponto de vista de detecção de possíveis tendências contínuas de elevação, bem como de sua data de início.

A complexidade da dinâmica e das teleconexões atmosféricas exige que a determinação das forçantes atuantes em um sistema seja realizada à luz das ciências físico/meteorológicas. Os resultados aqui apresentados devem ser interpretados como "ponto de partida" para tais investigações.

O primeiro passo na elaboração de um teste de aderência é a determinação de seu nível de significância. De acordo com WiLKs (2006), uma das formas de interpretar este nível é por meio da probabilidade de rejeitar-se Ho, sendo esta verdadeira. Essa falsa rejeição é denominada de erro tipo I e sua probabilidade, nível do teste, é usualmente representada por á. Erros tipo I são definidos em contraste aos erros tipo II, que ocorrem quando não há a rejeição de uma Ho falsa. A probabilidade do erro tipo II é usualmente denominada â.

É ilustrada na Figura 2 a relação entre o erro tipo I e tipo II em um teste conduzido ao nível á $=0,05$.

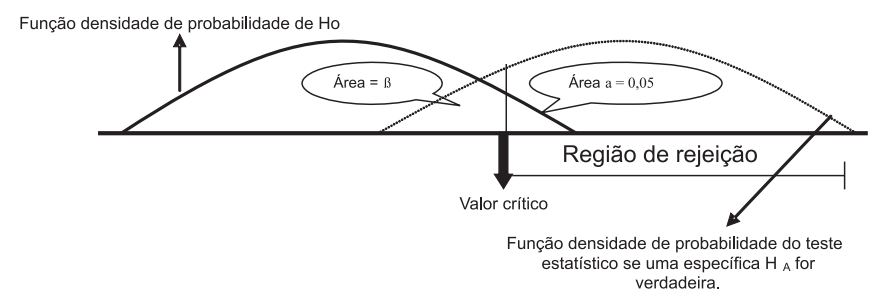

Figura 2. Relação entre o nível á de rejeição, correspondente à probabilidade á do erro tipo I (área á); e a probabilidade â do erro tipo II (área â). O eixo horizontal representa os possíveis valores do teste estatístico.
Segundo WiLKs (2006), seria extremamente interessante minimizar as probabilidades tanto do erro tipo I quanto do erro tipo II. Entretanto, nota-se na figura 2, ainda que o nível do teste seja ajustado, o que corresponde a mover o valor crítico para direita diminuindo o valor de á, ou para esquerda, aumentar o valor desse parâmetro á, a queda da probabilidade do erro tipo I acarreta na elevação da probabilidade do erro tipo II.

Apesar dessa dificuldade, muitas vezes, é importante examinar a variabilidade de â em um intervalo de possíveis $\mathrm{H}_{\mathrm{A}}$. Esse tipo de análise é citada por WILKS (2006) com o nome de rigor do teste (power of the test) e é normalmente realizada em termos do valor 1 - â em contraste a uma específica $\mathrm{H}_{\mathrm{A}}$. Na figura 2, o rigor do teste corresponde, para um dado $\mathrm{H}_{\mathrm{A}}$, a área à direita da distribuição amostral, excluindo-se a área relativa a â. A relação entre o rigor do teste e uma específica hipótese alternativa é chamada de "power function" e expressa a probabilidade de rejeitar-se Ho em função "do quanto a hipótese está errada". Esse fato justifica a escolha de um nível á mais elevado, $10 \%$, por exemplo, imprimindo melhor equilíbrio na probabilidade dos erros tipo I e II. Foram adotados os níveis 10\% e 5\% para a condução do teste Kolmogorov-Smirnov.

Na tabela 2, observa-se que as séries utilizadas podem ser consideradas provenientes de uma população com distribuição normal aos níveis de significância de $10 \%$ e $5 \%$. O valor calculado do teste permaneceu, em todas as séries, bastante abaixo dos patamares críticos. São ilustradas, na figura 3, as formas gráficas das FDPN para os períodos de 1917 a 1946, 1947 a 1976 e 1977 a 2006 relativas aos postos meteorológicos de Campinas e Piracicaba. O forte deslocamento à direita das FDPN, relativas aos períodos de 1917 a 1946, 1947 a 1976 e 1977 a 2006, evidencia o alto grau de elevação da série anual de temperatura mínima de Campinas, bem como sua característica linear, relatada em BLAIN et al. (2007). Observa-se aumento nos valores de Tmín em todas as classes de probabilidade.

Tabela 2. Valores do teste Kolmogorov-Smirnov (KS) para as séries anuais de temperatura mínima nas regiões de Campinas, Cordeirópolis/Limeira, Monte Alegre do Sul, Pindorama, Piracicaba e Ribeirão Preto, Estado de São Paulo, Brasil.

\begin{tabular}{lcccc}
\hline Local & Série & KS & V.Crítico $10 \%$ & V.Crítico 5\% \\
\hline Campinas & 1917 a 2006 & 0,0424 & 0,1286 & 0,1434 \\
Piracicaba & 1917 a 2006 & 0,0205 & 0,1286 & 0,1434 \\
Cordeirópolis / Limeira & 1951 a 2006 & 0,0377 & 0,1630 & 0,1817 \\
Monte Alegre do Sul & 1951 a 2006 & 0,0767 & 0,1630 & 0,1817 \\
Pindorama & 1951 a 2006 & 0,0648 & 0,1630 & 0,1817 \\
Ribeirão Preto & 1951 a 2006 & 0,0684 & 0,1630 & 0,1817 \\
\hline
\end{tabular}



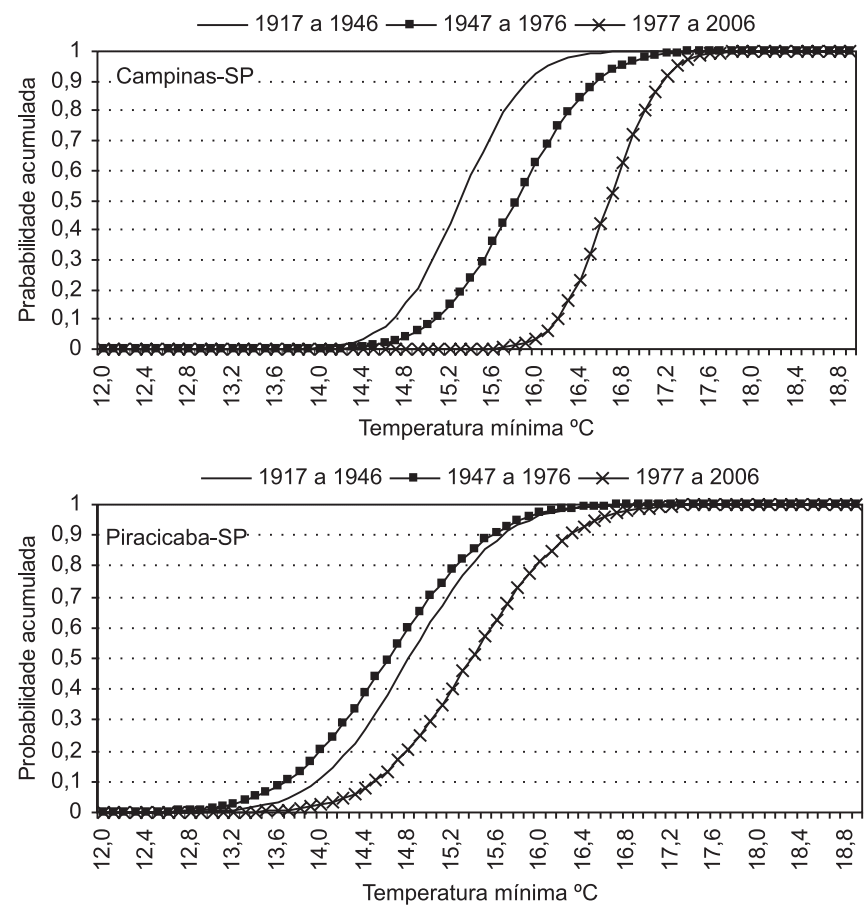

Figura 3. Formas gráficas das funções densidades de probabilidade, para os períodos de 1917 a 1946, 1947 a 1976 e 1977 a 2006, relativas aos postos meteorológicos de Campinas e Piracicaba, Estado de São Paulo, Brasil.

Em relação à série de Piracicaba, nota-se que, ao contrário do ocorrido em Campinas, no espaço amostral de 1947 a 1976 foram observados os menores valores de temperatura mínima. Este período coincide com uma fase fria da Oscilação Decadal do Pacífico (ODP), descrita em ZhANG et al. (1997), MANTUA et al. (1997) e ZHANG et al. (1998).

As influências dessa oscilação de escala multidecadal na variabilidade climática da América do Sul foram investigadas por ANDREOLI e KayAnO (2005) e KAYANO e ANDREOLI (2007). Nesses trabalhos, verifica-se que para a precipitação pluvial esse fenômeno age de maneira construtiva (destrutiva) quando o El Niño/Oscilação Sul (ENOS) e a ODP estão na mesma (oposta) fase.

Essa característica observada na localidade de Piracicaba indica a necessidade de investigações que relacionem a ODP à variabilidade da temperatura do ar no Estado de São Paulo. Em outro aspecto, nota-se que a provável influência de forçantes radioativas locais nos registros de temperatura mínima, coletados na estação meteorológica de Campinas, pode ter se sobreposto à influência de fatores meteorológicos de grande escala como a PDO, por exemplo. Torna-se, portanto evidente, a necessidade de isolar fatores locais antes que estudos de ordem global sejam realizados com base em séries de postos meteorológicos.
São demonstradas na figura 4 as séries anuais de Tmín e as regressões lineares simples dessas duas localidades.

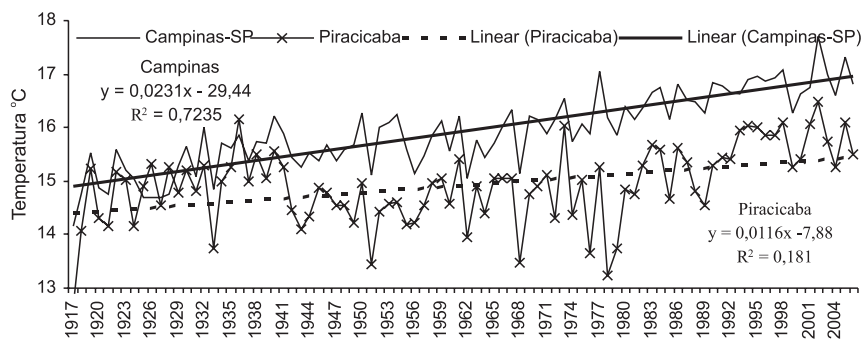

Figura 4. Séries anuais de temperatura mínima das localidades de Piracicaba e Campinas, Estado de São Paulo, Brasil.

Pelos valores da série histórica de Tmín de Campinas, no período entre 1917 a 2006 (Figura 4), por meio de análise de regressão simples, observa-se forte tendência de elevação. O modelo linear explica cerca de $72 \%$ desse aumento. Entretanto, assim como na figura 3, essa característica não é observada na localidade de Piracicaba. Nessa região, o método da regressão linear simples resultou em um coeficiente de deternimação $\left(R^{2}\right)$ próximo a 0,18 .

Dessa forma, considerando a proximidade geográfica entre esses dois postos, nota-se que a principal forçante radioativa da elevação linear dos valores de Tmín observados na série de Campinas parece estar mais ligada a fatores locais, como por exemplo à ilha de calor urbano, do que a fatores de grande escala, como por exemplo aquecimento global. São ilustrados na figura 5 os valores de $u(t n)$ e $u^{*}(t n)$ relativos à aplicação do MK para as séries de Campinas e Piracicaba.

$\mathrm{Na}$ figura 5 , verifica-se que a tendência de aumento dos valores de Tmín da série de Campinas é significativa, com início aproximado em 1968 . O ponto de intersecção entre $u(t n)$ e $u^{*}(t n)$ foi igual à 4,2 . Na localidade de Piracicaba, observa-se tendência de aumento não significativa com início aproximado em 1990. O ponto de intersecção entre $u(t n)$ e $u^{*}(t n)$, igual à 0,82 , permaneceu dentro do intervalo de confiança adotado. Com isso, verifica-se que a aplicação do teste de MK, parece também indicar que a elevação linear dos valores de Tmín observados na série de Campinas é mais influenciada por fatores locais do que por fatores de grande escala. A comparação entre as duas regiões, geograficamente bastante próximas, não revelou concomitância nas tendências contínuas de elevação da Tmín. Em outro aspecto, o intervalo temporal de aproximadamente 12 anos entre a data de mudança de local do posto e a intersecção entre $u(t n)$ e $u^{*}(t n)$, aliada às afirmações de Mello et al. (1994) relativas à série 
de Campinas, indica que as alterações na variabilidade dos valores dessa série, dificilmente estejam ligadas a essa mudança no local de coleta. São ilustradas na figura 6 as formas gráficas das FDPN para os períodos de 1951 a 1976 e 1977 a 2006 relativas aos postos meteorológicos de Pindorama, Monte Alegre do Sul, Cordeirópolis/Limeira e Ribeirão Preto.
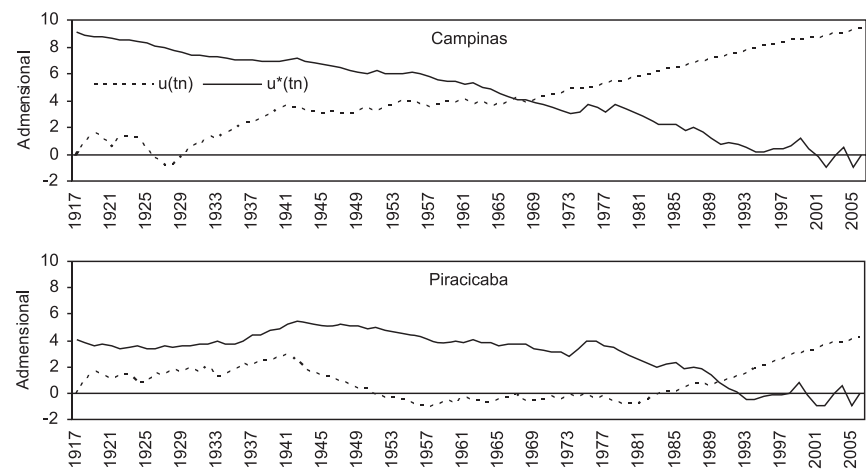

Figura 5. Valores de $\mathrm{u}(\mathrm{tn})$ e $\mathrm{u}^{*}(\mathrm{t})$ relativos à aplicação do teste de Mann-Kendall para as séries de Campinas e Piracicaba, Estado de São Paulo, Brasil.

Analisando-se a figura 6 e comparando-se os períodos de 1951 a 1976 e 1977 a 2006, nota-se que as séries de Cordeirópolis/Limeira e Ribeirão Preto indicam considerável tendência de elevação, demonstrada pelo deslocamento à direita das FDPN. Entretanto, essa característica não é observada nas regiões de Monte Alegre do Sul e, especialmente, de Pindorama. Nesta última série, há um ponto de intersecção nas duas curvas, constatando-se que em Pindorama valores de temperatura mínima anual superiores a $16,5^{\circ} \mathrm{C}$ revelam maiores probabilidades de ocorrência no período de 1951 a 1976 do que no período de 1977 a 2006.
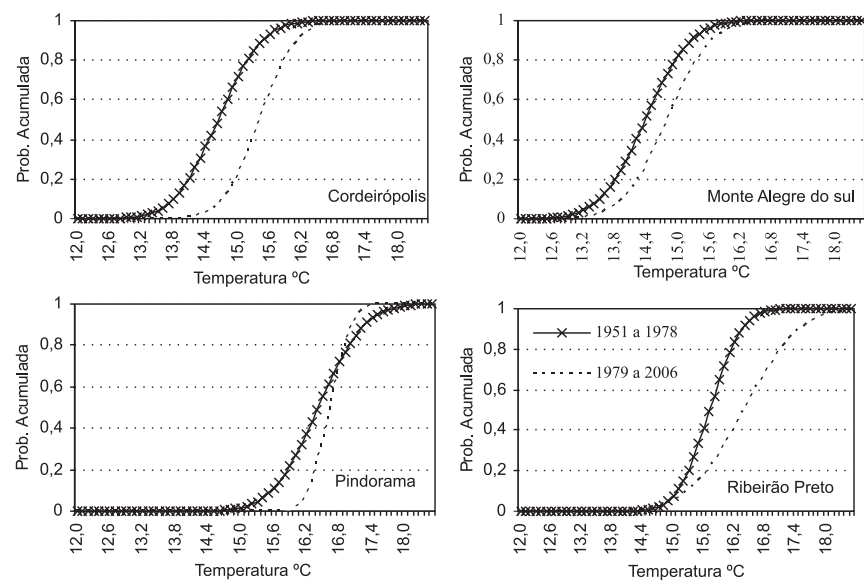

Figura 6. Formas gráficas das funções densidades de probabilidade, para os períodos de 1951 a 1976 e 1977 a 2006, relativas aos postos meteorológicos de Cordeirópolis/Limeira, Monte Alegre do Sul, Pindorama e Ribeirão Preto, Estado de São Paulo, Brasil.
São demonstradas na Figura 7 as séries anuais de Tmín das localidades de Pindorama, Monte Alegre do Sul, Ribeirão Preto e Cordeirópolis/Limeira.

Analisando-se a figura 7 , nota-se que nas regiões de Pindorama e Monte Alegre do Sul, por meio da regressão linear simples, não se observa tendência de aumento linear, e os coeficientes de determinação são, respectivamente, iguais a 0,10 e 0,07.

Nas localidades de Ribeirão Preto e Cordeirópolis/Limeira essa tendência é mais evidente, com o modelo linear explicando aproximadamente $43 \%$ e $53 \%$ da variabilidade da Tmín respectivamente. Na figura 8 são relacionados os valores de $u(t n)$ e $\mathrm{u}^{*}(\mathrm{tn})$ relativos à aplicação do teste de MK para as séries de Pindorama, Monte Alegre do Sul, Ribeirão Preto e Cordeirópolis/Limeira.

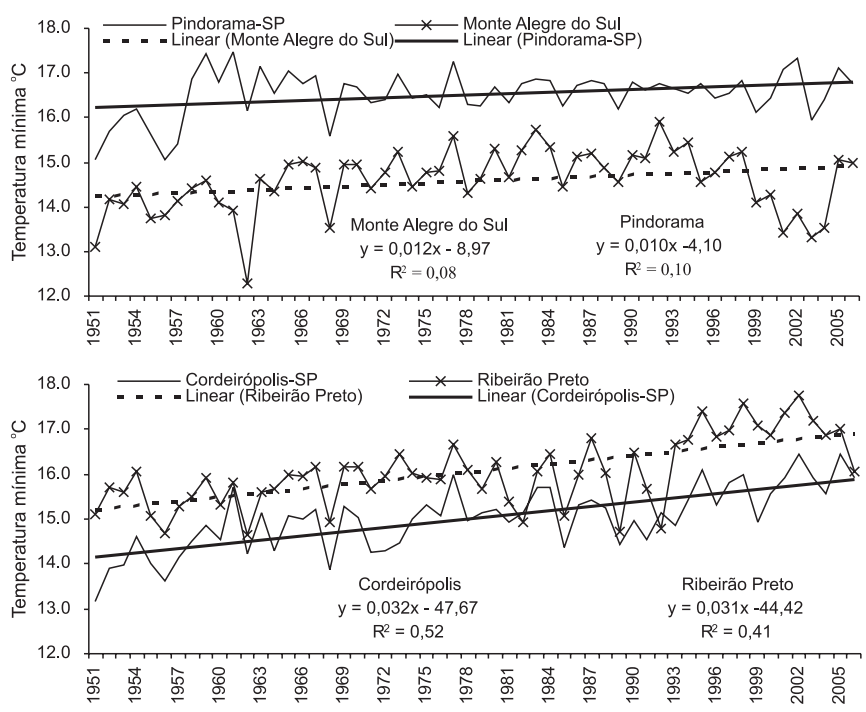

Figura 7. Séries anuais de temperatura mínima das localidades de Pindorama-SP, Monte Alegre do Sul, Ribeirão Preto e Cordeirópolis/Limeira do Estado de São Paulo.

Analisando-se a figura 8, nota-se que nas localidades de Cordeirópolis e Ribeirão Preto há tendências significativas e concomitantes de elevação nos valores de Tmín. Na série de Cordeirópolis/ Limeira a intersecção de $\mathrm{u}(\mathrm{tn})$ e de $\mathrm{u}^{*}(\mathrm{tn})$ ocorreu no valor 2,55 e em 1989.

Na série de Ribeirão Preto esta intersecção ocorreu no valor 2,80 em 1988. Entretanto, nas séries de Monte Alegre do Sul e Pindorama, pelo teste MK não se observou tendência de elevação, e a distribuição de probabilidade relativa aos valores de Tmín permaneceu constante caracterizando uma série aleatória simples. 

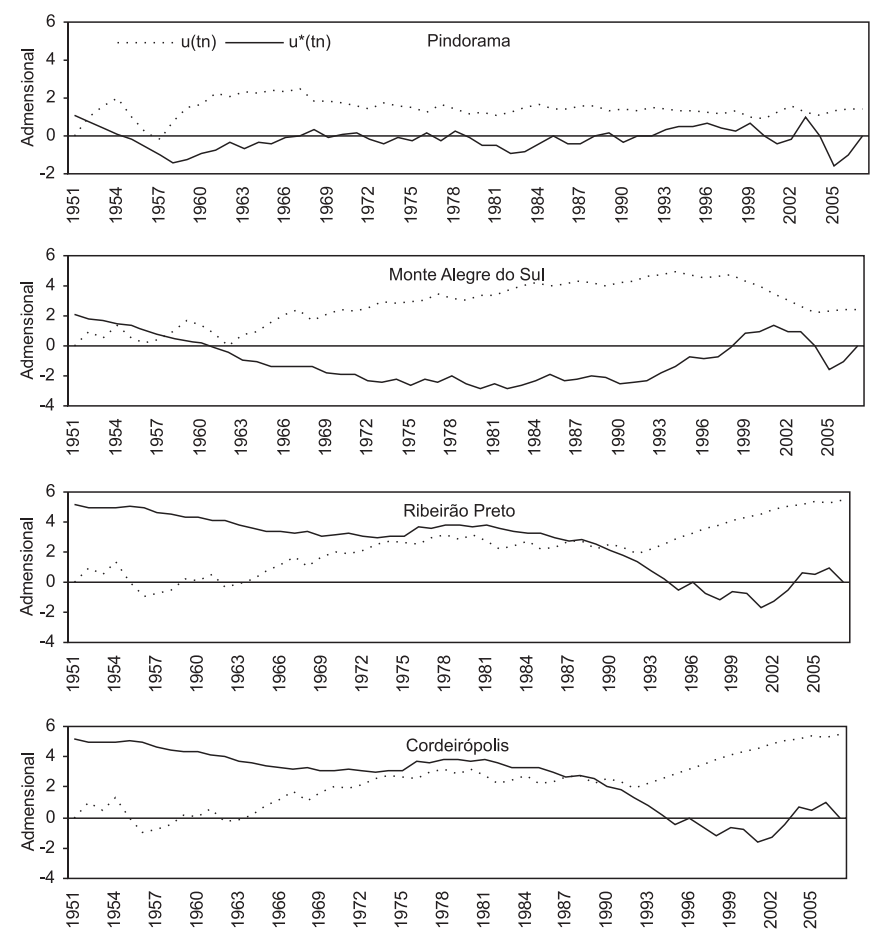

Figura 8. Valores de $\mathrm{u}(\mathrm{tn})$ e $\mathrm{u}^{*}(\mathrm{tn})$ do teste de MannKendall para as cidades de Pindorama, Monte Alegre do Sul, Ribeirão Preto e Cordeirópolis/Limeira, Estado de São Paulo, Brasil.

Ainda que nas regiões de Cordeirópolis/ Limeira e Ribeirão Preto tenha ocorrido elevação concomitante nos valores de Tmín, a inexistência dessa tendência nas localidades de Pindorama e Monte Alegre do Sul reforça a hipótese de que a principal forçante radioativa, causadora desse aumento linear nas séries analisadas, é relativa a fatores locais e não a fatores de escala global. Entretanto, ressalta-se que, devido à complexidade do tema aquecimento global, os resultados deste estudo não são conclusivos quanto à inexistência de alterações das influências de fenômenos globais nas séries de temperatura mínima do Estado de São Paulo. Contudo, esses resultados indicam a necessidade de avaliar e/ou isolar fatores locais antes que investigações de ordem global sejam realizadas em séries históricas de postos meteorológicos.

A comparação dos resultados observados nas localidades de Cordeirópolis/Limeira e Piracicaba demonstra também a complexidade do estudo das diversas forçantes físico/meteorológicas que atuam sobre o clima/tempo de uma região. Sob o ponto de vista de número de habitantes, a detecção de tendências significativas de elevação nas séries de Tmín das localidades de Campinas e Ribeirão Preto, cidades mais populosas do estudo, e sua ausência nos postos de Monte Alegre do Sul e Pindorama, menos populosas, indicam correlação positiva entre o crescimento populacional e aumento da Tmín, observado em estações meteorológicas de superfície. Entretanto, na região de Limeira/Cordeirópolis, com aproximadamente 300.000 habitantes, houve tendência significativa de elevação na série de Tmín; na região de Piracicaba, com aproximadamente 366.000 habitantes, não foram observadas tendências significativas de elevação.

É interessante ressaltar que o vale do Rio Piracicaba promove o acúmulo do ar frio noturno na região da cidade de Piracicaba. Essa característica orográfica, além de explicar o fato de as temperaturas mínimas do município de Campinas serem superiores às da região de Piracicaba, apesar da proximidade geográfica e da menor altitude dessa última localidade, pode ser a causa de não terem sido observadas tendências significativas de elevação nos valores de Tmín relativos à região contida nesse vale.

\section{CONCLUSÕES}

1. Das seis séries de temperatura mínima anual analisadas no trabalho, em apenas três (Campinas, Cordeirópolis/Limeira e Ribeirão Preto) há tendências significativas de elevação ocorrida de forma temporalmente distinta entre essas localidades. Nas demais localidades, não houve detecção de elevação significativa nos valores de temperatura mínima média anual. Dessa forma, fatores de escala local parecem sobreporem-se a possíveis fatores de escala global, como principais forçantes radioativas no aumento médio dos valores dessa variável meteorológica nessas seis regiões do Estado de São Paulo. Observa-se, portanto, a necessidade de avaliar e/ou isolar fenômenos locais, relativos à urbanização, por exemplo, antes que investigações de ordem global sejam realizadas em séries históricas de postos meteorológicos.

2. Pelos dados de Tmín relativos aos postos de Cordeirópolis/Limeira, Ribeirão Preto e, especialmente, Campinas, verificam-se contrastes significativos com as regiões rurais desses municípios. Esse fato pode diminuir a consistência de um zoneamento agrometeorológico que utilize tais séries. Investigações a esse respeito devem ser realizadas.

\section{AGRADECIMENTO}

Os autores agradecem à Prof. ${ }^{a}$ Dr. ${ }^{a}$ Mary Toshie Kayano pelos ensinamentos sobre investigação científica e estatística climatológica. 


\section{REFERÊNCIAS}

ANDREOLI, R.V.; KAYANO, M.T. ENSO-related rainfall anomalies in South America and associated circulation features during warm and cold Pacific decadal oscillation regimes. International Journal of Climatology, v.25, p.2017-2030, 2005.

ASSIS, F.N.; ARRUDA, H.V.; PEREIRA, A.R. Aplicações de estatística à climatologia. Pelotas: Universidade Federal de Pelotas, Universitária, 1996. 161p.

ASSIS, J.P.; NETO, D.D.; MANFRON, P.A.; MARTIN, G.S.; SPAROVEK, G.; TIMM, L.C. Ajuste de séries históricas de temperatura e radiação solar global diária às funções densidade de probabilidade normal e log-normal, em Piracicaba-SP. Revista Brasileira de Agrometeorologia, Santa Maria, v.12, n.1, p.113-121, 2004.

ASTOLPHO, F.; CAMARGO, M.B.P.; PEDRO JÚNIOR, M.J.; FILHO, W.J.P.; BARDIN, L. Probabilidades mensais e anuais de ocorrência de temperaturas mínimas do ar adversas à agricultura na região de Campinas (SP), de 1891 a 2000. Bragantia, v.63, p.141-147, 2004.

BLAIN, G.C.; ROLIM, G.; SENTELHAS, P.C.; LULU, J. Variabilidade temporal da temperatura do ar em Campinas, SP: Análise de tendências e mudanças climáticas. In: CONGRESSO BRASILEIRO DE AGROMETEOROLOGIA, 15., 2007, Aracaju. Anais... Aracaju: Sociedade Brasileira de Agrometeorologia, 2007.

CAMARGO, M.B.P.; PEDRO JÚNIOR, M.J.; ALFONSI, R.R.; ORTOLANI, A.A.; BRUNINI, O. Probabilidade de ocorrência de temperaturas mínimas absolutas mensais e anuais no Estado de São Paulo. Bragantia, v.52, p.161-168, 1993.

FREITAS, E.D.; SILVA-DIAS, P.L. Alguns efeitos de áreas urbanas na geração de uma ilha de calor. Revista Brasileira de Meteorologia, v.20, p.355-366, 2005.

GOOSSENS, C.; BERGER, A. Annual and seasonal climatic variations over the northern hemisphere and Europe during the last century. Annales Geophysicae, v.4, p.385-400, 1986.

ICHINOSE, T.; SHIMODOZONO, K.; HANAKI, K. Impact of anthropogenic heat on urban climate in Tokyo. Atmospheric Environment, v.33, p.3897-3909, 1999.

IPCC. The Physical Science Basis. Contribution of Working Group I to the Fourth Assessment Report of the Intergovernmental Panel on Climate Change. In: HOUGHTON, J.T. (Ed.). Climate Change. New York: Cambridge University Press, 2007.

KAYANO, M.T.; ANDREOLI, R.V. Relations of South American summer rainfall interannual variations with the Pacific Decadal Oscillation. International Journal of Climatology, v.27, p.531-540, 2007.

LOMBARDO, M.A. Ilha de calor da metrópole paulistana. 1984. 210p. Tese (Doutorado) - Faculdade de Filosofia, Letras e Ciências Humanas-USP, São Paulo.
MANTUA, N.J.; HARE, S.R.; ZHANG, Y.; WALLACE, J.M.; FRANCIS, R.C. A Pacific interdecadal climate oscillation with impacts on salmon production. Bulletin of the American Meteorological Society, Boston, v.78, p.1069-1079, 1997.

MELLO, M.H.A.; PEDRO JÚNIOR, M.J.; ORTOLANI, A.A.; ALFONSI, R.R. Chuva e temperatura: cem anos de observações em Campinas. Campinas: Instituto Agronômico, 1994. 48p.

MORAES, J.M.; PELLEGRINO, G.; BALLESTER, M.V.; MARTINELLI, L.A.; VICTORIA, R.L. Estudo preliminar da evolução temporal dos componentes do ciclo hidrológico da bacia do Rio Piracicaba. In: SIMPÓSIO BRASILEIRO DE RECURSOS HÍDRICOS, 11., 1995, Recife. Anais... Recife: Associação Brasileira de Recursos Hídricos, 1995.

MORETTIN, P.L.; BUSSAB, W.O. Estatística básica. São Paulo: Saraiva, 2003. 526p.

OKE, T.R. Boundary layer climates. London: Routledge, 1987. $435 p$.

ROLIM, G.; CAMARGO, M.B.P.; LANIA, D.G. Classificação climática de Köppen e de Thornthwaite e sua aplicabilidade na determinação de zonas agroclimáticas para o Estado de São Paulo. Bragantia, v.66, p.711-720, 2007.

SANSIGOLO, C.A. Distribuições de extremos de precipitação diária, temperatura máxima e mínima e velocidade do vento em Piracicaba, SP (1917-2006). Revista Brasileira de Meteorologia, São José dos Campos, v.23, p.341-346, 2008.

SNEYERS, R. Sur 1'Analyse Statistique des Séries d'Observations. Genève: Organisation Météorologique Mondial, 1975. 192p.

WILKS, D.S. Statistical methods in the atmospheric sciences. 2.ed. San Diego: Academic Press, 2006. 629p.

ZHANG, Y.; WALLACE, J.M.; BATTISTI, D. ENSO-like interdecadal variability: 1900-93. Journal of Climate, London, v.5, p.1004-1020, 1997.

ZHANG, Y.; NORRIS, J.; WALLACE, J.M. Seasonality of largescale atmosphere-ocean interaction over the North Pacific. Journal of Climate, London, v.11, p.2473-2481, 1998. 